\title{
AMPA/kainate glutamate receptor antagonists prevent posttraumatic osteoarthritis
}

\author{
Cleo S. Bonnet,, ${ }^{1,2}$ Sophie J. Gilbert,, Emma J. Blain, ${ }^{1,2}$ Anwen S. Williams, ${ }^{2,3}$ and Deborah J. Mason ${ }^{1,2}$ \\ ${ }^{1}$ School of Biosciences, ${ }^{2}$ Biomechanics and Bioengineering Research Centre Versus Arthritis, and ${ }^{3} \mathrm{~S} c h o o l$ of Medicine, \\ Cardiff University, Cardiff, United Kingdom.
}

\begin{abstract}
Musculoskeletal disorders represent the third greatest burden in terms of death and disability in the developed world. Osteoarthritis is the single greatest cause of chronic pain, has no cure, and affects 8.5 and 27 million people in the UK and US, respectively. Osteoarthritis is most prevalent in older people, but as it commonly occurs after joint injury, young people with such injuries are also susceptible. Painful joints are often treated with steroid or hyaluronic acid (HA) injections, but treatments to prevent subsequent joint degeneration remain elusive. In animals, joint injury increases glutamate release into the joint, acting on nerves to cause pain, and joint tissues to cause inflammation and degeneration. This study investigated synovial fluid glutamate concentrations and glutamate receptor (GluR) expression in injured human joints and compared the efficacy of GluR antagonists with current treatments in a mouse model of injury-induced osteoarthritis (ACL rupture). GluRs were expressed in the ligaments and meniscus after knee injury, and synovial fluid glutamate concentrations ranged from 19 to $129 \mu \mathrm{M}$. Intra-articular injection of NBQX (GluR antagonist) at the time of injury substantially reduced swelling and degeneration in the mouse ACL rupture model. HA had no effect, and Depo-Medrone reduced swelling for 1 day but increased degeneration by $\mathbf{5 0} \%$. Intra-articular administration of NBQX modified both symptoms and disease to a greater extent than current treatments. There is an opportunity for repurposing related drugs, developed for CNS disorders and with proven safety in humans, to prevent injury-induced osteoarthritis. This could quickly reduce the substantial burden associated with osteoarthritis.
\end{abstract}

Conflict of interest: CSB and DJM have obtained patents (US 9,918,986, EP3016636 and ZL201480047577.6) with respect to the technology described herein.

Copyright: (c) 2020, Bonnet et al. This is an open access article published under the terms of the Creative Commons Attribution 4.0 International License.

Submitted: October 17, 2019

Accepted: May 29, 2020

Published: July 9, 2020.

Reference information: /CI Insight. 2020;5(13):e134055.

https://doi.org/10.1172/jici.

insight.134055.

\section{Introduction}

Musculoskeletal disorders represent the third greatest burden in terms of death and disability in the developed world, causing $21.3 \%$ of the total years lived with disability globally (1). Osteoarthritis (OA) affects 8.5 million people in the UK $(2,3)$ and 27 million people in the US (4), causing substantial physical, psychological, and socioeconomic burdens (4-7). At least $12 \%$ of the current OA cases were caused by prior joint injury $(8,9)$, so-called posttraumatic OA (PTOA). For example, anterior cruciate ligament rupture (ACLr) causes knee OA in $50 \%-90 \%$ of patients 5-15 years later (10-12). This example, along with other joint injuries (13-16), potentially inflicts OA at a young age. Since there are no disease-modifying treatments for OA, which usually manifests after substantial joint damage has already occurred, effective interventions at the time of injury offer an opportunity for prevention of PTOA.

Current treatments for unresolved joint pain, early OA, and joint injuries include intra-articular (i.a.) steroid (e.g., Depo-Medrone) or hyaluronic acid (HA), but few preclinical studies test whether these or other agents given i.a. at the time of joint injury influence OA progression. Intra-articular amnion/ chorion membrane 24 hours after meniscal transection surgery in rats (17), dexamethasone immediately following surgical drill injury in rabbits (18), and IL-1 receptor antagonist (IL-1RA) given immediately after articular fracture in mice $(19,20)$ all reduced joint degeneration to some extent. Of these, IL-1RA has progressed to phase II trials for prevention of PTOA (ClinicalTrials.gov NCT02930122), whereas dexamethasone (ClinicalTrials.gov NCT02318433) is undergoing phase I trials for this indication.

Glutamate signaling contributes to pain, inflammation, bone remodeling, and degradation in arthritis $(21,22)$. Synovial fluid (SF) glutamate concentrations increase approximately 54-fold in patients with arthritis compared with those in cadaveric non-OA samples (23) and double after inflammatory arthritis 
induction (24) and ACL transection-induced OA (25) in rats in vivo. Increased glutamate released during arthritis acts on nerves to drive peripheral pain, with locally delivered glutamate receptor (GluR) antagonists inhibiting pain behavior in carrageenan-induced arthritis (26), monosodium-iodoacetate-induced arthritis (27), antigen-induced arthritis (AIA) (22), and inflammatory pain in arthritic mice (28). Glutamate also regulates inflammation. SF glutamate concentrations in patients with rheumatoid arthritis (RA) correlate with chemokine levels and GluR agonists induce TNF- $\alpha$ release in RA synovial cells (29) and increase TNF- $\alpha$ and RANTES release by human synovial cells (30). Human RA synoviocytes express functional $\alpha$-amino-3-hydroxy-5-methyl-4-isoxazolepropionic acid (AMPA) and kainate GluRs, which regulate IL-6 release (31), an essential mediator of arthritic joint degradation (32). Glutamate also influences pathological processes. We have shown that a single i.a. injection of NBQX (AMPA/kainate antagonist) in rat AIA significantly reduced knee swelling by 33\%, histological synovial inflammation by $34 \%$, and joint degeneration scores by $27 \%$, exceeding the efficacy of etanercept, infliximab, and methotrexate (22). Others have shown that memantine (N-methyl-D-aspartate [NMDA] GluR antagonist, intraperitoneally every 12-24 hours) reduces synovitis and bone erosions in collagen-induced arthritis (33).

Previously, we showed that subchondral bone remodeling, an early driver of OA pathology (34-36), was substantially reduced by NBQX treatment in rat AIA (22). Spontaneous OA animal models show that subchondral bone remodeling precedes cartilage damage (36-40) and correlates with cartilage lesions (41). In humans, ACLr leads to subchondral bone remodeling that precedes cartilage destruction (34), with bone bruises occurring in $80 \%$ of patients within 2 weeks of ACLr that later associate with cartilage damage (42). Thus, using AMPA/kainate GluR antagonists following trauma to inhibit these early pathologic changes in bone may reduce subsequent cartilage degradation.

We hypothesized that glutamate drives OA initiation following acute injury and that GluR antagonists could prevent OA symptoms and joint degeneration. Glutamate signals through ionotropic (iGluRs: AMPA, kainate, NMDA) and metabotropic (mGluR1-8) GluRs expressed by all joint tissues (21, 22). Drugs targeting these receptors have been thoroughly investigated for disorders of the nervous system, such as epilepsy, motor neuron disease, stroke, and migraine, and offer a rich opportunity for quick translation to new indications.

Although AMPA/kainate GluR antagonists are disease modifying in AIA (22), and SF glutamate concentrations increase in human and animal arthritis (23-25), it is not known whether glutamate concentrations vary at the time of traumatic injury in humans or animals or whether GluR antagonists given at the time of injury are protective. Here, we measure SF glutamate concentrations after human ACL injury and demonstrate that i.a. NBQX treatment given at the time of injury was disease modifying in the mouse ACLr model, where controlled loading of the joint ruptures the ligament in the absence of surgery (43). NBQX efficacy exceeded that of Depo-Medrone and HA; when these were given in the same way at doses used clinically, they were either ineffective (HA) or increased joint degeneration (Depo-Medrone). AMPA/kainate receptor antagonists, originally developed for epilepsy, migraine, and pain and already safety tested in humans, represent a promising translational opportunity for repurposing for prevention of injury-induced OA.

\section{Results}

Glutamate concentration is similar in $O A$ and after injury and GluRs, are expressed in knee tissues from injured patients. Mean glutamate concentrations were highest in RA SF $(62.3 \pm 8.5 \mu \mathrm{M})$, followed by ACLr $(55.2 \pm 4.98$ $\mu \mathrm{M})$, meniscal tear $(43.1 \pm 14.42 \mu \mathrm{M})$, and OA $(39.2 \pm 9.44 \mu \mathrm{M})$ (Figure $1 \mathrm{~A}, n=5 /$ group, except ACLr, where $n=27$, and RA, where $n=3$ ). Glutamate concentrations varied significantly with time after ACL injury (ANOVA, $P=0.03)$; they were greater at $0-20(58.3 \pm 5.84 \mu \mathrm{M}, P<0.05, n=12)$ and $21-100(70.64$ $\pm 10.32 \mu \mathrm{M}, P<0.01, n=8)$ weeks after injury, compared with 100-500 (34.9 $\pm 7.79 \mu \mathrm{M}, n=7)$ weeks after injury (Figure 1B, Tukey's). Across the whole patient cohort, SF glutamate concentrations showed a trend toward reduced concentrations with increasing age (Figure 1C, Pearson's correlation coefficient $-0.330, P=$ 0.053). There were no differences in male and female SF glutamate concentrations (Figure 1D).

AMPAR2 and kainate-1 (KA1) were expressed in ACL fibroblasts and meniscal chondrocytes from patients with ACLr or meniscal injury (Figure 1, E-H).

NBQX reduces inflammation in ACLr PTOA and is more effective than HA or steroid. ACLr significantly increased knee swelling in $\mathrm{H}_{2} \mathrm{O}$ vehicle-treated mice $(P<0.001$, general linear model [GLM]), and this was significantly reduced by NBQX treatment $(P<0.001$, GLM) (Figure 2A). On days 1, 2, 3, and 7 after ACLr, knee swelling in vehicle-treated mice was significantly greater than day 0 swelling $(P<0.001$, Tukey's), whereas NBQX-treated mice only showed significant increases on day $1(P=0.007$, Tukey's) and 
A

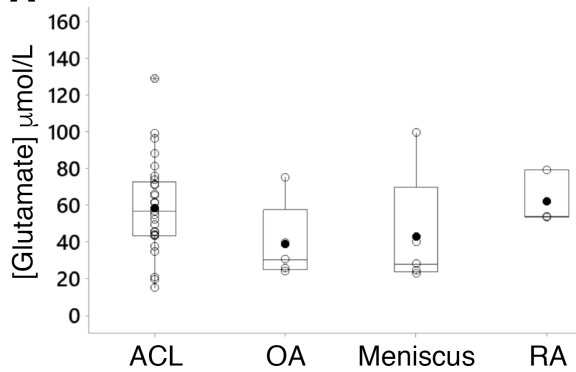

C

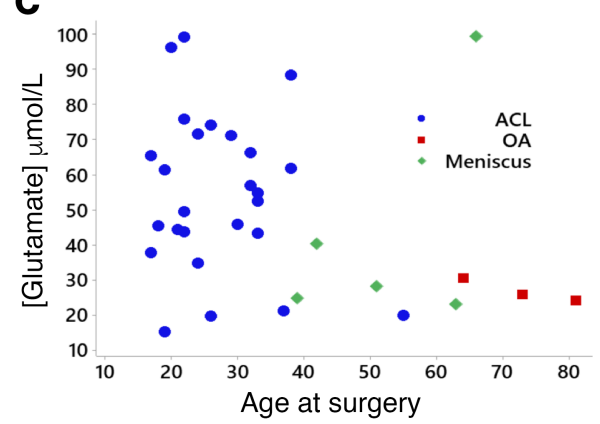

B

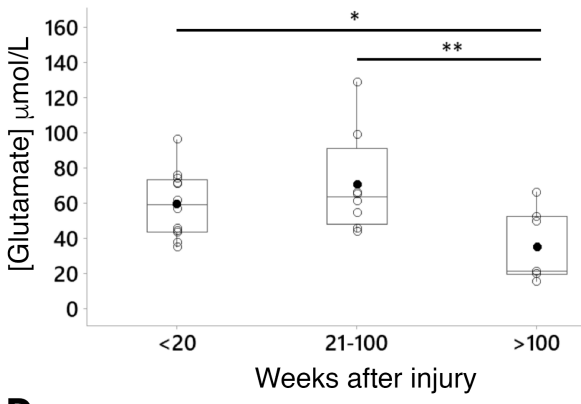

D

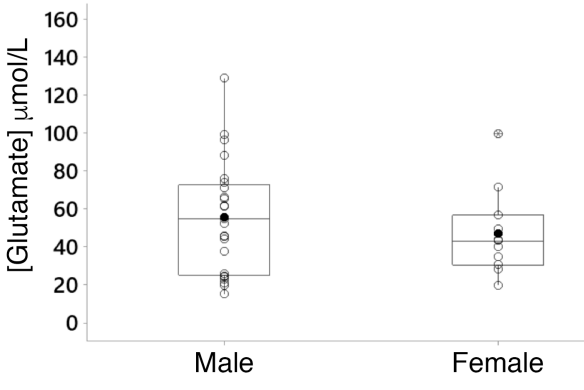

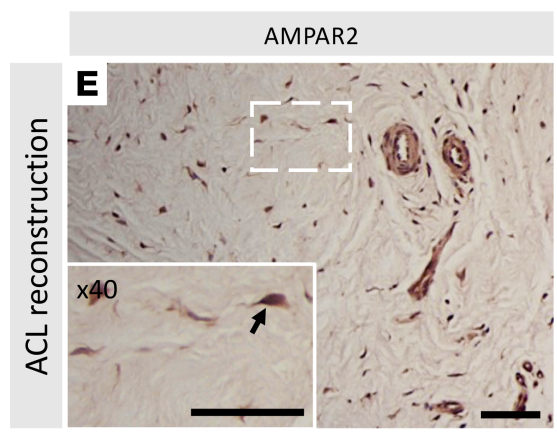
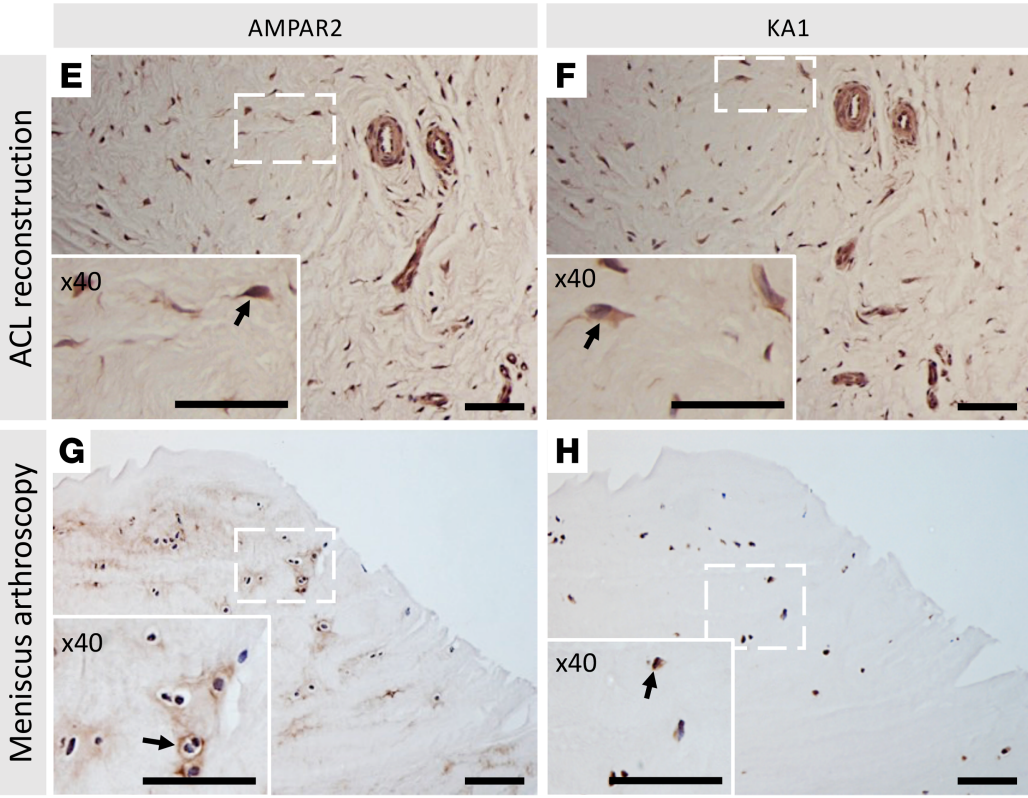

Figure 1. Glutamate concentrations and receptors in patients. (A) Glutamate was detectable in synovial fluid samples from $27 \mathrm{ACL}$ reconstruction, 5 osteoarthritic total knee replacement (TKR), 5 meniscal arthroscopy, and 3 rheumatoid arthritis (RA) patients. (B) Glutamate concentrations were significantly greater at 0-20 $(n=12)$ and 21-100 $(n=8)$ weeks after ACL injury, compared with 100-500 ( $n=7)$ weeks after injury (ANOVA $P=0.03$, Tukey's post hoc: $\left.{ }^{*} P<0.05,{ }^{* *} P<0.01\right)$. (C) Synovial fluid glutamate concentrations appear to decrease with increasing age, although not significantly (Pearson's correlation coefficient $-0.330, P=0.053)$. (D) There are no differences in male $(n=25)$ and female $(n=11)$ synovial fluid glutamate concentrations. (E-H) Matched tissue samples from patients in A were used for AMPAR2 and kainate-1 (KA1) immunohistochemistry. AMPAR2 and KA1 receptors were expressed in ACL fibroblasts ( $E$ and $\mathbf{F}$ ) and meniscal chondrocytes $(\mathbf{G}$ and $\mathbf{H})$ from patients with $A C L$ and meniscus injuries, respectively. Areas within dashed boxes are presented at higher magnification in insets. Arrows indicate positive staining. Scale bars: E-H $(100 \mu \mathrm{m}) ; 50 \mu \mathrm{m}$ (insets). Original magnification, $\times 40(\mathbf{E}-\mathbf{H}$, insets). Data are presented as box plots (representing interquartile range, median, and all data points, including mean, which is indicated by a crossed circle) in $\mathbf{A}, \mathbf{B}$, and $\mathbf{D}$.

day $2(P=0.023$, Tukey's) compared with day 0 (Figure 2A). NBQX treatment significantly reduced knee swelling after ACLr on days 1 and 2 compared with vehicle-treated mice (by $44 \%$ and $45 \%, P=0.007$ and $P=0.02$, respectively, Tukey's) (Figure 2A). Steroid (Depo-Medrone) treatment significantly reduced knee swelling on day 1 compared with vehicle ( $P=0.001$, Tukey's) and HA ( $P=0.017$, Tukey's) (Figure 2B). 
Knee swelling in steroid- ( $P<0.01$, Tukey's), HA- $(P<0.001$, Tukey's), and vehicle-treated $(P<0.001$, Tukey's) mice remained significantly higher than day 0 (before ACLr) measurements until 14 days after rupture (Figure 2B).

At day 21, synovial hyperplasia and infiltrate induced by ACLr was similar for NBQX, HA, and their respective vehicle controls (Kruskal Wallace with Mann-Whitney post hoc test) (Figure 2, C-E). Steroid treatment increased mean inflammation score, although not significantly (Figure 2, D and E).

$N B Q X$ does not influence pain-related behavior in ACLr PTOA. Lameness score reduced over time for both NBQX- and vehicle-treated ACLr mice $(P<0.001, \mathrm{GLM})$; day 7 scores were significantly lower than day 1 scores, and NBQX treatment had no significant effect on lameness compared with vehicle (Figure 2F). A significant interaction between time and treatment $(P<0.001$, GLM) revealed that NBQX-treated ACLr mice were more lame on days 1-3 but were not lame on days 14 and 21 compared with vehicle-treated ACLr mice. Irrespective of time, steroid and HA treatment reduced the lameness score compared with treatment with saline (treatment $P<0.001$, GLM; $P<0.001$, steroid vs. saline; $P$ $<0.001$, HA vs. saline, Tukey's), although no significant differences were found on individual days and mice remained lame until day 14 (Figure $2 \mathrm{G}$ ).

$N B Q X$ reduces joint degradation in ACLr PTOA whereas steroid increases damage. After ACLr, NBQX treatment reduced cartilage and bone pathology by $29 \%$, from a severity score of $31.3 \pm 1.23$ to $22.2 \pm 2.72(P$ $<0.001$, 2-sample $t$ test), although not to naive values (7.6 \pm 0.67$)$ (Figure $3 \mathrm{~A})$. Individual parameters of the scoring system revealed that ACLr caused substantial loss of cartilage (13.65 \pm 0.6$)$, proteoglycan loss (11.4 \pm 0.5$)$, and subchondral bone remodeling $(6.41 \pm 0.39)$, whereas NBQX reduced these by $26 \%(10.05$ $\pm 1.12, P=0.001), 25 \%(8.5 \pm 0.96, P=0.004)$, and $43 \%$ (3.65 $\pm 0.74, P<0.001)$, respectively (2-sample $t$ tests, Figure 3C). Significant reductions in joint severity score caused by NBQX treatment were seen in both the medial (29\%, from $23.56 \pm 1.07$ to $16.63 \pm 2.65, P<0.05$, Mann-Whitney test) and lateral ( $28 \%$, from $7.77 \pm 0.4$ to $5.58 \pm 0.32, P<0.001,2$-sample $t$ test) sides of the joint (Figure 3D).

Steroid (Depo-Medrone) (43.79 \pm 2.5 ) treatment significantly increased joint degradation by $52 \%$ or $53 \%$ compared with HA $(28.8 \pm 3.03)$ or saline vehicle treatment $(28.71 \pm 3.49)$, respectively $(P<0.05$, 1-way ANOVA) (Figure 3B).

Representative (median score) images reveal that ACL rupture caused subchondral bone thickening, osteophytes, and cartilage loss, particularly on the medial side of the joint, that were substantially reduced by NBQX treatment (Figure 3E). Joints treated with HA appeared similar to vehicle controls; however, steroid treatment caused severe bone loss down through the growth plate on both medial and lateral sides as well as the formation of large chondrophytes (developing osteophytes) and ectopic bone (Figure 3E).

Double dose of $N B Q X$ is most effective at preventing joint degradation in the ACLr model. Both the number of injections ( 1 vs. 2 vs. $3, P=0.024$ ) and the type of treatment (NBQX vs. vehicle, $P=0.02$ ) significantly affected joint degeneration after ACLr (2-factor GLM, Supplemental Figure 1A; supplemental material available online with this article; https://doi.org/10.1172/jci.insight.134055DS1). Two injections significantly reduced joint severity scores compared with a single injection following ACLr $(P<0.02, \mathrm{GLM})$ (Supplemental Figure 1A). Two i.a. doses of NBQX (at the time of rupture and 24 hours later) reduced the mean knee severity score to $10.35 \pm 2.6$ compared with 1 dose $(19.4 \pm 3.5)$ or 3 doses of NBQX (24.1 $\pm 4.0)$ as well as 2 doses of vehicle control $(21.7 \pm 4.2)$ (Supplemental Figure $1 \mathrm{~A})$. The knee severity score following 2 doses of NBQX was restored to that of control, uninjured knees, whereas all other treatments remained significantly higher (Supplemental Figure 1A). When broken down into parameters, 2 doses of NBQX significantly reduced scores compared with 2 doses of vehicle ( $P=0.002$, GLM) (Supplemental Figure 1B). Two doses of NBQX reduced OA changes by $51 \%$, bone changes by $78 \%$, and proteoglycan loss by $42 \%$ (Supplemental Figure 1B). Knee swelling and lameness over time, and day 21 histological inflammation, were not affected by the dosing regimen (Supplemental Figure 1, C-E).

NBQX treatment alters GluR expression in ACLr PTOA. To determine whether AMPA and kainate GluRs are expressed in PTOA, reveal potential target tissues, and determine whether expression patterns changed with NBQX treatment, we immunolocalized GluRs in each treatment group at disease end stage (21 days) (Figure 4). Expression patterns are summarized in Table 1. It is important to note that due to the progression of OA after ACLr, surface chondrocytes are often not present, and it is not possible to distinguish which cartilage zones remain.

AMPAR2 stained the synovial lining strongly in both NBQX-treated (Figure 4C) and vehicle-treated (Figure 4G) ACLr mice but was less abundant in intact ACL (Figure 4K). AMPAR2 staining of 
A

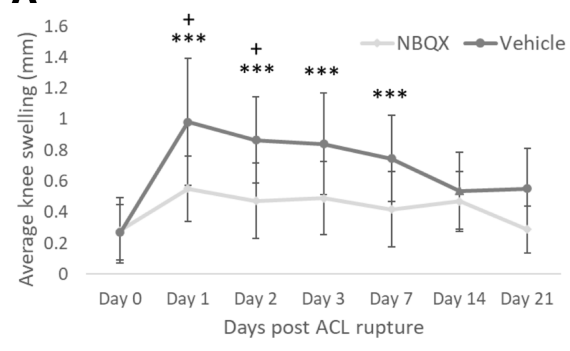

C

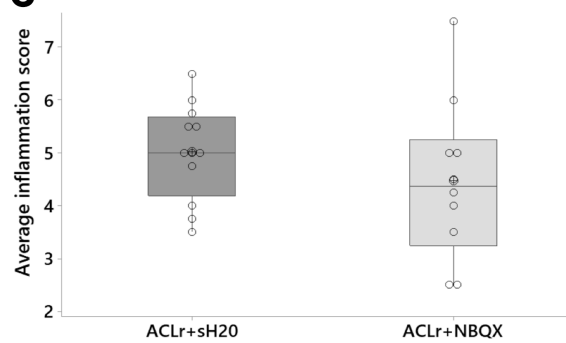

B

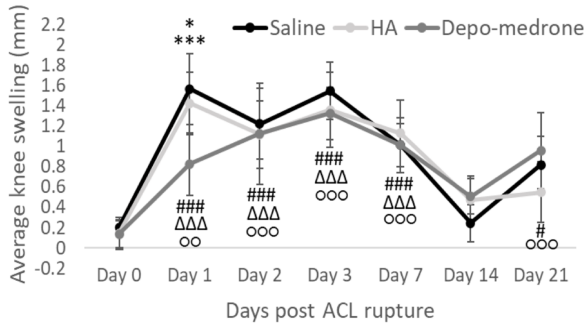

D

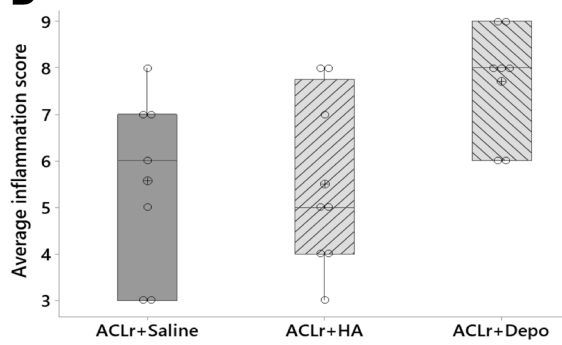

E

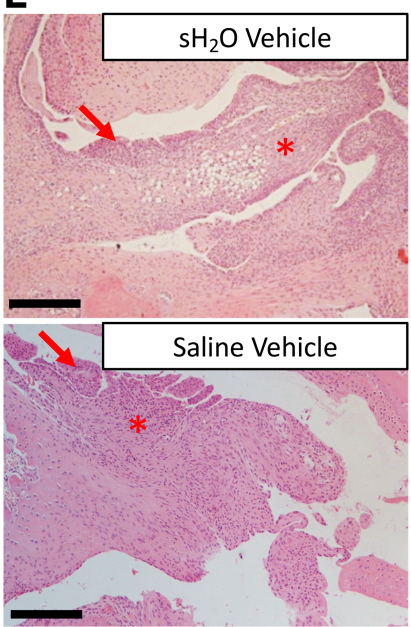

$\mathbf{F}$

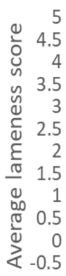

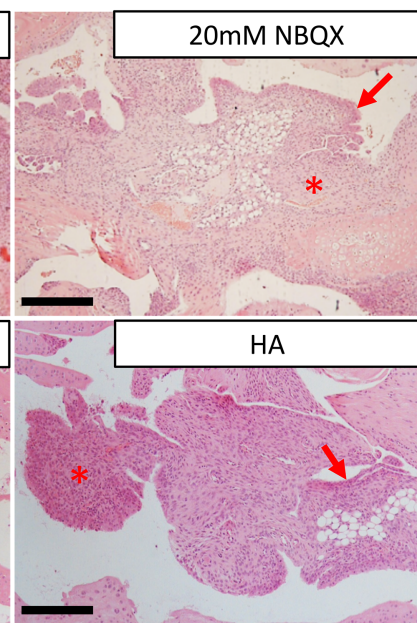

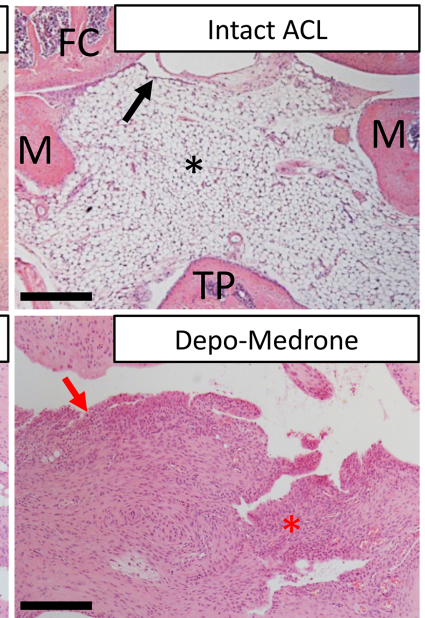

$\mathbf{G}$
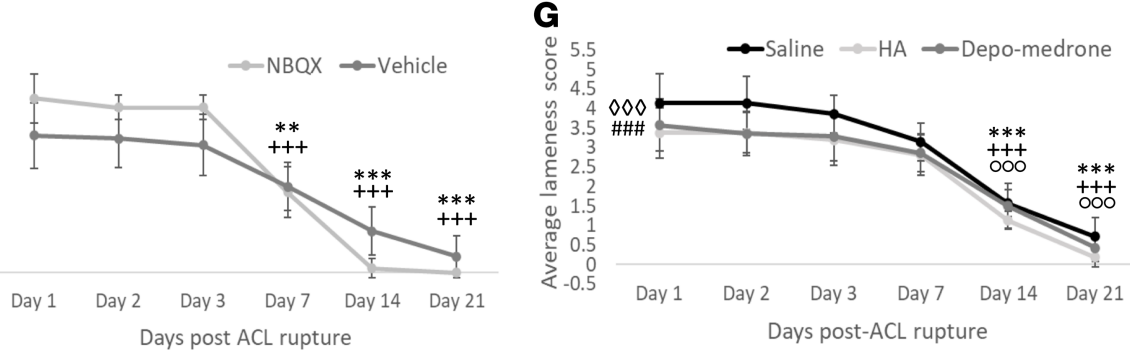

Figure 2. Knee swelling, histological inflammation, and pain behavior in ACLr mice treated with $20 \mathrm{mM}$ NBQX versus vehicle (water) and HA or Depo-Medrone versus vehicle (saline). (A) On days 1, 2, 3, and 7 after ACLr, knee swelling in vehicle-treated mice was significantly greater than day 0 swelling ${ }^{* * * *} P<0.001$, Tukey's), whereas NBQX-treated mice showed no significant increase compared with day 0 swelling. NBQX treatment significantly reduced knee swelling on days 1 and 2 compared with that in vehicle-treated mice $\left({ }^{+} P<0.05,{ }^{+} P<0.01\right.$, Tukey's). (B) Steroid (Depo-Medrone) treatment significantly reduced knee swelling on day 1 compared with vehicle ( ${ }^{* *} P<0.001$, Tukey's) and HA treatment $\left({ }^{*} P<0.05\right.$, Tukey's). Knee swelling in steroid- $\left({ }^{\circ 0} P<0.001,{ }^{\circ} P<0.01\right.$, Tukey's), HA- $\left({ }^{\circ \Delta \Delta} P\right.$

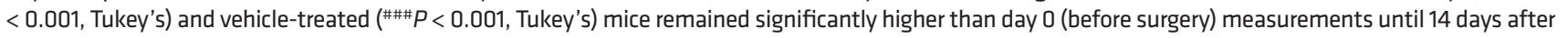
rupture. At day 21, the synovial inflammation score was similar for NBQX- (C), HA- (D), and vehicle-treated mice, whereas steroid treatment increased the mean inflammation score, although this increase was not significant (D). (E) Contralateral knees with intact ACL displayed no signs of inflammation. Intact ACL mice displayed normal synovial lining, 2- to 4-cells thick (black arrow), with underlying adipose tissue (black asterisk). ACLr with vehicle treatment induced synovial hyperplasia (red arrows) and infiltrate (red asterisks) that were also present following NBQX, HA, and steroid treatment. FC, femoral condyle; TP, tibial plateaux; M, meniscus. Scale bars: $100 \mu \mathrm{m}$. (F) The lameness score reduced over time for both NBQX-treated and vehicle-treated control ACLr mice; day 7, 14, and 21 measurements were significantly lower than those from day $1\left({ }^{+++} P<0.001, \mathrm{NBQX}\right.$; ${ }^{* * *} P<0.001,{ }^{* *} P<0.01$ vehicle, Tukey's). (C) Steroid and HA treatment reduced the lameness score (independent of time) compared with saline (CLM for treatment $P<0.001$, Tukey's post hoc test: ${ }^{000} P<0.001$, steroid vs. saline; \#\#\# $P<0.001$, HA vs. saline), although no significant differences were found on individual days. B y day 14 , the lameness score was significantly lower for saline, $H A$, and steroid compared with day 1 scores $\left({ }^{* * *} P<0.001\right.$, saline; ${ }^{+++} P<0.001, \mathrm{HA} ;{ }^{000} \mathrm{P}<0.001$, steroid, Tukey's). Data are presented as mean $\pm \mathrm{SD}$ in $\mathbf{A}, \mathbf{B}, \mathbf{F}$, and $\mathbf{G}$ and box plots (representing interquartile range, median, and all data points, including mean, which is indicated by a crossed circle) in C and D. $20 \mathrm{mM} \mathrm{NBQX}(n=10)$, vehicle (water, $n=15)$, HA ( $n=8)$, Depo-Medrone $(n=7)$, vehicle (saline, $n=7)$. 
A

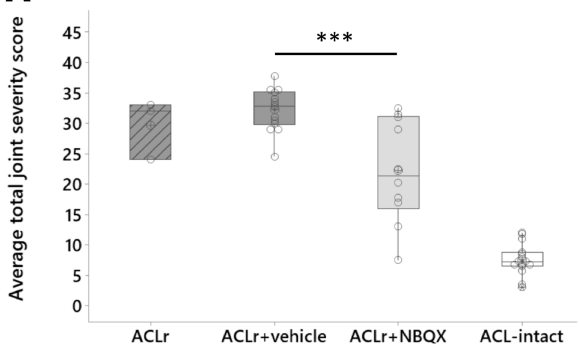

C

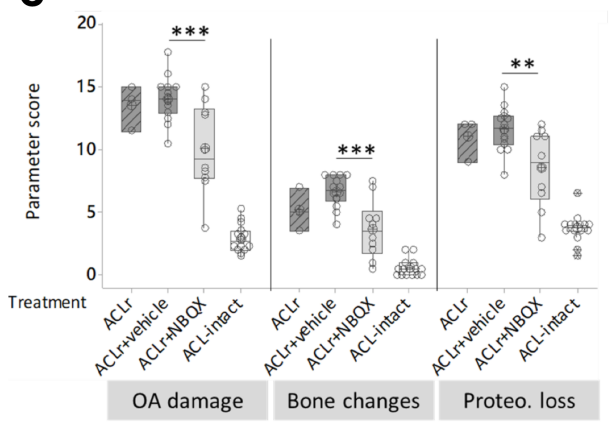

E

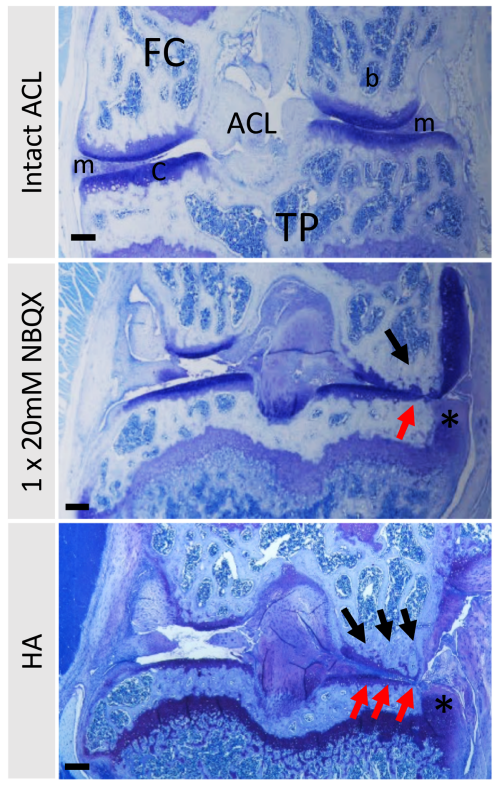

B

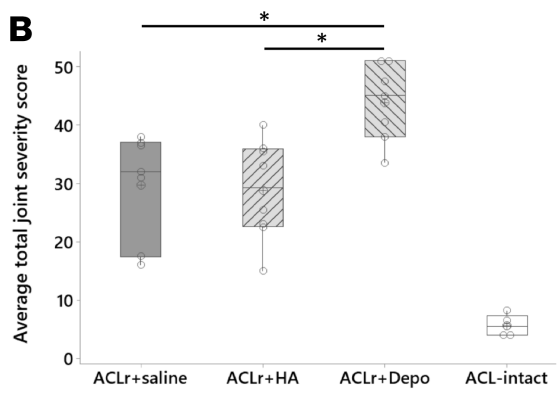

D
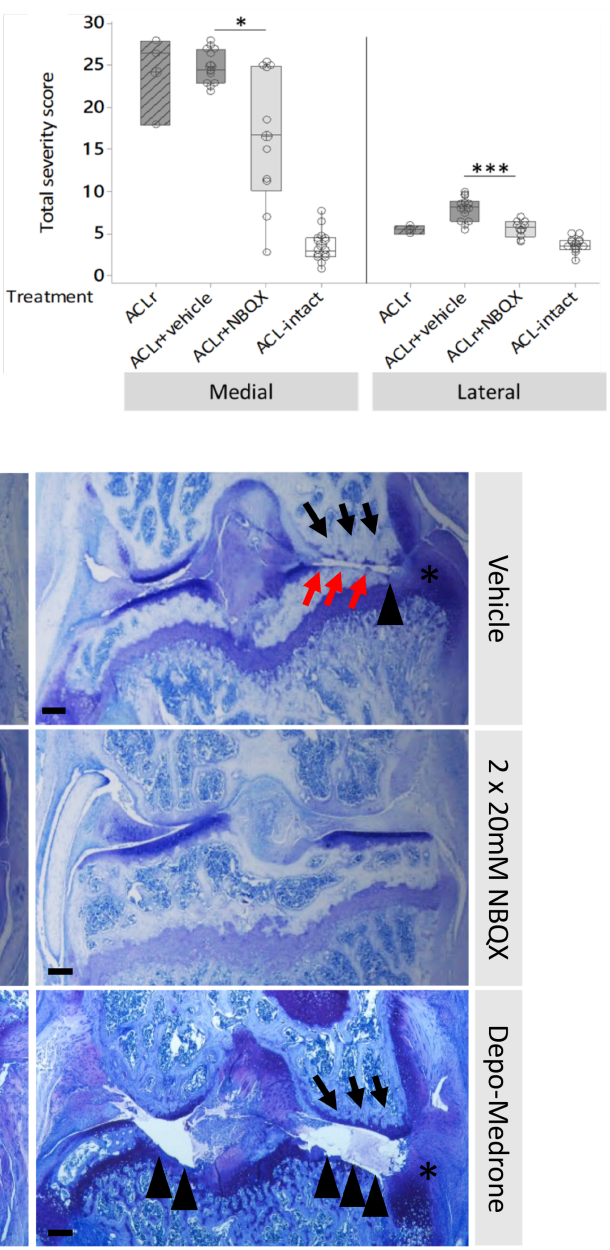

Figure 3. Histological knee joint severity in ACLr mice treated with $20 \mathrm{mM}$ NBQX versus vehicle (water) and HA or DepoMedrone versus vehicle (saline). (A) At day 21, NBQX treatment significantly reduced the joint severity score compared with vehicle ( ${ }^{* *} P<0.001,2$-sample $t$ test). (B) Steroid (Depo-Medrone) treatment significantly increased joint degradation by approximately $50 \%$ compared with $\mathrm{HA}$ or saline vehicle treatment $\left({ }^{*} P<0.05\right.$, 1-way ANOVA with Tukey's post hoc). (C) When broken down into parameters, NBQX significantly reduced cartilage loss (OA damage, ${ }^{* * *} P<0.001$ ), proteoglycan loss ( $\left.{ }^{* *} P<0.01\right)$, and bone changes (***P $<0.001,2$-sample $t$ tests). (D) Significant reductions in joint severity score caused by NBQX treatment were seen in both the medial ${ }^{*} P<0.05$, Mann-Whitney test) and lateral ( ${ }^{* *} P<0.001,2$-sample $t$ test) sides of the joint. (E) Healthy bone (b), covered by a smooth articular cartilage (c), was clearly present in knees with an intact $A C L$; however, following $A C L r$ (with vehicle), severe cartilage loss is evident (red arrows), in conjunction with bone thickening and remodeling (black arrows) and chondrophyte formation (asterisks). Joint damage was sometimes so severe, cartilage and bone loss occurred down to the growth plate (black arrowhead). A single i.a. injection of NBQX reduced cartilage (red arrow) and bone changes (black arrow) and joints retained more structural integrity. Two injections of NBQX maintained joint structure similar to that seen in intact ACL samples. HA caused similar effects to vehicle, with severe cartilage loss (red arrows), bone thickening and remodeling (black arrows) and chondrophyte formation (asterisks). However, intra-articular Depo-Medrone was highly damaging to the joint, causing bone loss down through the growth plate (black arrowheads) in both the medial and lateral compartments and the formation of large chondrophytes (asterisk). FC, femoral condyle; TP, tibial plateaux; $\mathrm{ACL}$, anterior cruciate ligament; $\mathrm{m}$, meniscus. Scale bars: $50 \mu \mathrm{m}$. Data are presented as box plots (representing interquartile range, median, and all data points, including mean, which is indicated by a crossed circle). $20 \mathrm{mM}$ NBQX $(n=10)$, vehicle (water, $n=15), \mathrm{HA}(n$ $=8)$, Depo-Medrone $(n=7)$, vehicle (saline, $n=7)$.

chondrocytes was present throughout the cartilage in all 3 groups (Figure 4, A, B, E, F, I, and J). Osteocytes stained for AMPAR2 in NBQX-treated ( 50\%) (Figure 4D) and vehicle-treated $(\sim 75 \%)$ (Figure $4 \mathrm{H}$ ) ACLr mice, whereas only approximately $20 \%$ of osteocytes were positive in intact ACL (Figure 4L). Bone-lining cells stained strongly for AMPAR2 in vehicle-treated ACLr mice (Figure 4H), but AMPAR2 was less abundant after NBQX treatment (Figure 4D) and in intact ACL (Figure 4L).

KA1 stained the synovial lining in vehicle-treated ACLr mice (Figure 4S) but was not detected after NBQX treatment (Figure 4O) or in intact ACL (Figure 4W). KA1 stained chondrocytes in NBQX-treated ACLr mice (Figure 4, M and N) and intact ACL (Figure 4, U and V) but not vehicle-treated mice (Figure 4, Q and R). KA1 was not expressed in osteocytes in any groups (Figure 4, P, T, and X). Bone-lining cells 
showed weak KA1 staining after NBQX (Figure 4P) and vehicle (Figure 4T) treatment of ACLr mice, but KA1 expression appeared less abundant in intact ACL (Figure 4X).

AMPAR2 and KA1 staining was abundant in ruptured ACL from vehicle- (Figure 4, CC-FF) and NBQX-treated (Figure 4, Y-BB) ACLr mice, but KA1 staining was almost absent in intact ACL (Figure 4, GG-JJ, and Supplemental Figure 1, F-I). Significantly less positive KA1 staining was seen in intact ACL compared with ruptured ACL following 2 doses of either vehicle or NBQX (Supplemental Figure 1I).

\section{Discussion}

We report glutamate concentrations in human injured knee joint fluids for the first time to our knowledge. Patients with ACLr $(55 \mu \mathrm{M})$ or meniscal tear $(43 \mu \mathrm{M})$ had similar mean SF glutamate concentrations to those with OA $(39 \mu \mathrm{M})$, within reported ranges of patients with OA $(266 \mu \mathrm{M}$, range $0-530 \mu \mathrm{M})$ and RA $(326$ $\mu \mathrm{M}$, range 4-608 $\mu \mathrm{M})$ and exceeding ranges in postmortem nonarthritic humans $(6.25 \mu \mathrm{M}$, range $0.82-22$ $\mu \mathrm{M})$ and living nonarthritic rabbits $(4.23 \mu \mathrm{M})$ and rats $(2.72-5.91 \mu \mathrm{M})(23-25)$. Glutamate concentrations varied over time after ACL injury and were greatest before 100 weeks after injury. Mean SF concentrations ranged from $58 \mu \mathrm{M}$ at $0-20$ weeks and $71 \mu \mathrm{M} 21-100$ weeks after ACL rupture to $35 \mu \mathrm{M}$ at $>100$ weeks after injury. This temporal elevation in SF glutamate concentrations, along with our observation that AMPA and kainate GluRs are expressed after knee injury, and in arthritic joints (22), provides evidence that GluR antagonists may be an appropriate acute intervention in human PTOA. Both temporal and patient-specific variability in SF glutamate concentrations may reveal an opportunity for therapeutic targeting.

Our previous work indicated that AMPA and kainate GluRs on human synoviocytes regulated IL-6 release, an essential mediator of joint degeneration in inflammatory arthritis (31), and that NBQX intervention in inflammatory arthritis reduced pain, inflammation, and degeneration (22). We have recently described a mouse model of joint injury, where a single controlled load ruptures the ACL noninvasively, inducing very early inflammatory effects, including increased IL-6 expression (43). We therefore assessed the protective effect of $20 \mathrm{mM} \mathrm{NBQX}$ administered i.a. at the time of acute injury in the mouse ACLr model, as a mimic of acute joint injury $(43,44)$.

A single i.a. NBQX treatment after ACLr reduced knee swelling by up to approximately $45 \%$ through the 7 days following injury and reduced the joint severity score, representing cartilage and bone pathology, by $29 \%$. NBQX significantly reduced all components of the joint severity score but was most effective in reducing the bone score by $43 \%$, in keeping with our previous studies showing its control of bone changes in rat AIA (22). Intra-articular injection ensures bioavailability of the drug in the joint and controls dosing, but small molecules, such as NBQX, rapidly diffuse (0.23-hour half-life in humans; refs. 45). Two doses of NBQX (at the time of rupture and 24 hours later) reduced the joint severity score by $48 \%$, restoring values to those of uninjured knees. The 2-dose efficacy may reflect the intervention window in the ACLr model, as 3 doses (days 0,1 , and 7 after rupture) were less effective. However, repeated i.a. injections may increase inflammation and mask protective effects of longer-term treatments.

There are surprisingly few reports of other agents given i.a. acutely at the time of injury. In surgical models, i.a. delivery of dehydrated amnion/chorion membrane given 24 hours after surgery (17) appeared protective but was not conventionally scored, whereas 4 repeated i.a. IL-1RA injections (6 mg per 40- $\mu$ l injection) reduced cartilage histopathology after ACL transection in rats (46). Corticosteroid data are contradictory and have been reported to damage joint structures $(47,48) ; 1$ i.a. injection of dexamethasone $(0.5 \mathrm{mg} / \mathrm{kg})$ immediately following surgical drill injury in rabbits reduced Mankin score by approximately $40 \%$ (18), whereas a 0.5 -mg i.a. injection of dexamethasone given 7 days after surgery increased joint degradation in rabbit PTOA (49). Studies reporting the effect of i.a. drugs at the time of injury in nonsurgical PTOA are limited to those on IL-1RA (20). IL-1RA, which has progressed to human trials, given immediately after injury in a mouse articular fracture model of PTOA, improved mean degeneration scores by $30 \%$, restoring them to control values $(19,20)$. The disease-modifying effect of 1 i.a. injection of NBQX matched that of IL-1RA, and 2 doses exceeded its efficacy.

No studies have tested i.a. injections of corticosteroids or HA at the time of injury in nonsurgical rodent models of OA, even though these drugs are often used in painful, injured joints in humans. Therefore, we compared efficacy of NBQX with i.a. steroid (Depo-Medrone) or HA used at equivalent i.a. doses in mice as recommended in humans. Following ACLr, NBQX reduced knee swelling by approximately $45 \%$ over days $1-7$, whereas HA had no effect and Depo-Medrone reduced knee swelling by $50 \%$ on day 1 only. NBQX increased the lameness score by approximately $25 \%$ over days $1-3$ but prevented lameness after day 7, whereas Depo-Medrone and HA treatment reduced lameness scores by approximately $17 \%$ 


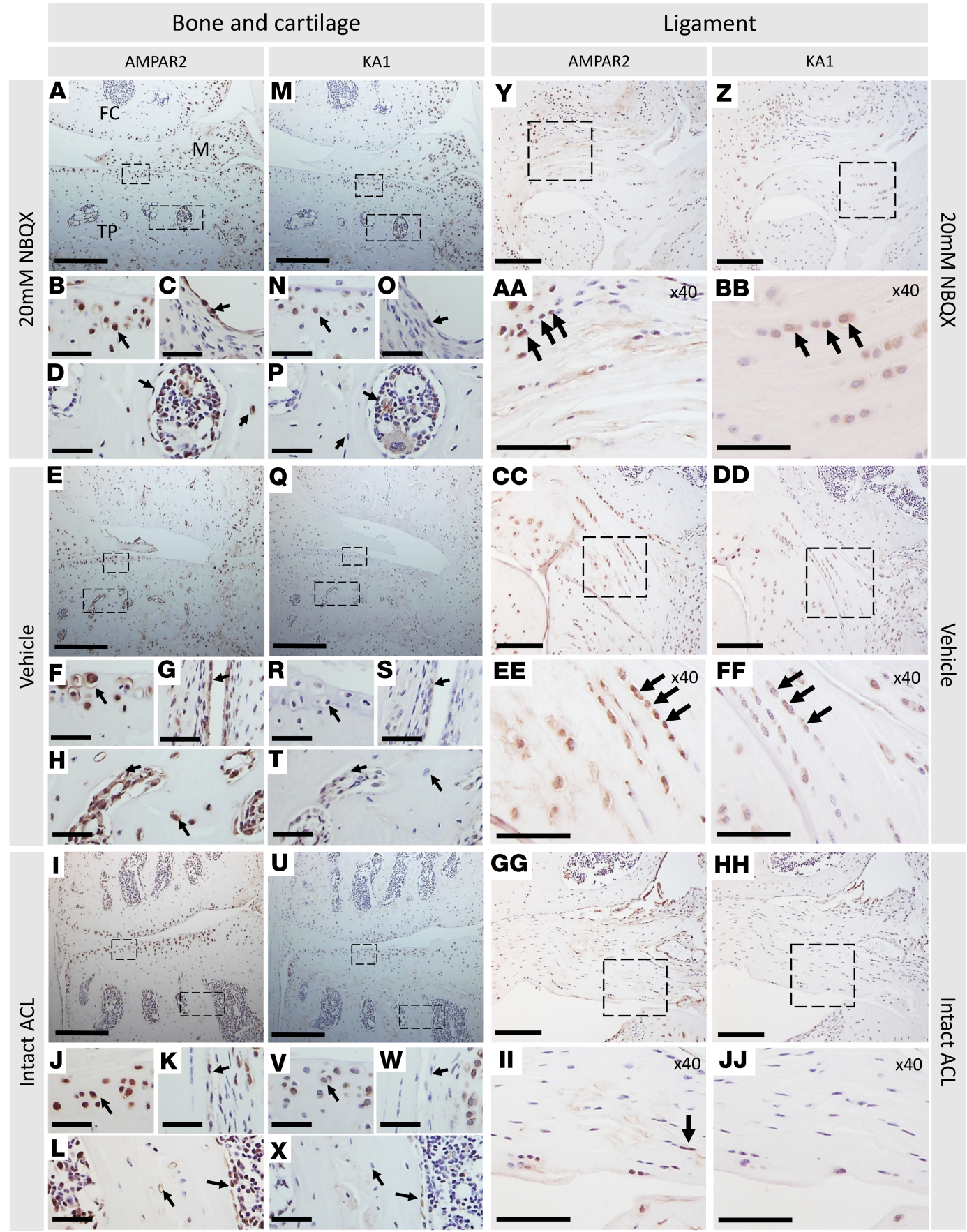

Figure 4. GluR expression in bone, cartilage, and ligament in the ACL rupture model. Strong AMPAR2 staining of synovial lining after NBQX (C) and vehicle (G) treatment was less abundant in intact ACL (K). AMPAR2 stained chondrocytes throughout cartilage under all conditions (A, B, E, F, I, and J). AMPAR2 stained osteocytes after NBQX (D) and vehicle (H) treatment, but fewer osteocytes were positive in intact ACL (L). AMPAR2 stained bone-lining cells after vehicle treatment (H) but was less abundant after NBQX treatment (D) and in intact ACL (L). KA1 stained synovial lining after vehicle treatment (S) but was not detected after NBQX treatment (0) or in intact ACL (W). KA1 stained chondrocytes after NBQX treatment ( $\mathbf{M}$ and $\mathbf{N}$ ) and in intact $A C L(\mathbf{U}$ and $\mathbf{V})$ but not after vehicle treatment $(\mathbf{Q}$ and $\mathbf{R})$. KA1 was not expressed in osteocytes (P, $\mathbf{T}$, and $\mathbf{X})$. KA1 weakly stained bone-lining cells after NBQX $(\mathbf{P})$ and vehicle $(\mathbf{T})$ treatment but was less abundant in intact ACL (X). AMPAR2 and KA1 staining was abundant in ACL fibroblasts from vehicle-treated ACL ruptured mice (CC-FF) but was less abundant in NBQX-treated mice (Y-BB) and almost absent in intact ACL (GG-JJ). Positive staining is indicated by black arrows. FC, femoral condyle; TP, tibial plateau; M, meniscus. Scale bars: $50 \mu \mathrm{m}$ (B-D, F-H, J-L, N-P, R-T, V-X, AA, BB, EE, FF, II, and IJ); $100 \mu \mathrm{m}$ (A, E, I, M, Q, U, Y, Z, CC, DD, GC, and HH). 
Table 1. AMPAR2 and KA1 protein expression in healthy mouse stifle and after ACL rupture with vehicle or NBQX treatment

\begin{tabular}{|c|c|c|c|c|c|c|}
\hline & \multicolumn{3}{|c|}{ AMPAR2 } & \multicolumn{3}{|c|}{ KA1 } \\
\hline & & Vehicle & NBQX & & Vehicle & NBQX \\
\hline Chondrocytes (clusters) & + & + & + & + & - & + \\
\hline Osteocytes & $+/-$ & ++ & + & - & - & - \\
\hline Osteoblasts/bone-lining cells & + & ++ & + & - & $+/-$ & $+/-$ \\
\hline
\end{tabular}

-, absent; +/-, weak; +, present; ++, abundant.

compared with vehicle but mice remained lame until day 14. Finally, NBQX reduced joint degeneration scores from $29 \%$ ( 1 dose) to $48 \%$ ( 2 doses), whereas a single dose of HA had no effect on degeneration, and Depo-Medrone significantly increased the joint severity score by $50 \%$ compared with saline control. The degenerative effects of Depo-Medrone are consistent with reports of its dose-dependent deleterious effects on cartilage morphology, histology, and cell viability both in vitro and in vivo (48) and the limited evidence of beneficial effects of i.a. injections of steroids in humans (50). Therefore, NBQX was more effective at reducing swelling and degeneration than steroid and HA after ACLr in mice.

A plethora of drugs have been developed to antagonize GluRs, some of which have passed phase I safety trials when used systemically (51). AMPA and kainate GluR antagonists have been tested predominantly for CNS diseases, such as epilepsy, with perampanel approved by EMA and FDA for epilepsy treatment. Although some of these drugs, taken orally as a daily medication, have been associated with side effects, most are safe and tolerated. Intra-articular injection into the joint acutely at the time of injury will reduce sustained bioavailability of the drug in the brain, which is likely to reduce side effects.

There are a number of limitations to this study. The SF glutamate concentrations after ACL rupture were only measured in 27 patients, reducing the power of detecting associations with time since injury, and although significant differences were observed with time, we did not measure glutamate concentrations for healthy controls. While mouse ACLr models are widely accepted PTOA models (52), limitations include thin articular cartilage, no zonal organization of cartilage, ossified meniscal cartilage, altered joint mechanics, and no closure of growth plates. We used day 21 after ACL rupture for data collection on endpoint OA disease and matched this to longitudinal measurements of in vivo changes in swelling and lameness. Further studies including earlier time points would allow for the timeline of OA progression to be compared among treatment groups. Although our previous work suggests that glutamate may be acting on the arthritic joint via IL-6 signaling (22, 31, 43), our current study lacks a mechanistic correlation with IL-6, and further in vitro/ex vivo studies are required to address this. Finally, the NBQX concentrations used in these experiments $(20 \mathrm{mM})$ may elicit nonspecific effects on other GluRs within the joint (51), and further studies are required to establish the minimum effective dose and optimum dose regime.

In conclusion, SF glutamate concentrations vary following knee injury in humans, and i.a. administration of the AMPA and kainate GluR antagonist NBQX administered at the time of injury was more effective at reducing swelling and degeneration than current treatments for joint pain and early OA. Repurposing clinic-ready AMPA/kainate GluR antagonists with proven safety in humans would substantially reduce the burden of injury-induced OA and represents a major advance that could quickly address this unmet need.

\section{Methods}

Patients. Matched SF, ACLs, and menisci were obtained from randomly selected patients undergoing total knee replacement (TKR) for OA ( 2 men, 1 woman), ACL reconstruction ( 2 men, 1 woman), or meniscal arthroscopy ( 2 women). Additional SF was obtained from 2 patients after ACL reconstruction ( 2 men), 3 patients after meniscal arthroscopy ( 2 men, 1 woman), 2 patients after OA TKR ( 1 man, 1 woman), and 2 patients after RA TKR (1 man, 1 woman). To examine SF glutamate levels at different times after ACL injury, 24 additional patients (18 men, 6 women) were identified with known injury date and sample extraction date. Age, sex, BMI, and time since injury were recorded (Supplemental Table 1). 
Animals. Procedures were performed according to Home Office and ARRIVE guidelines (53) on male C57/BL/6J mice (12 weeks old) housed in groups of 5 (Envigo; 12-hour light/12-hour dark cycles, ad libitum food and water). Animals were randomly assigned to the various experimental groups and treatments and randomly distributed among cages. The data were collected and processed randomly. All animal studies were blinded. Investigators did not know which animals had received which treatments during data acquisition and subsequent scoring of samples and were unaware of the allocated treatment at the time of induction of PTOA. Animals were monitored for welfare (weight, limping, posture) daily in the first week and weekly thereafter.

ACLr model. Custom built cups (54) were used to hold the right knee of anesthetized mice in flexion with a preload of $0.5 \mathrm{~N}$ before the application of a $12-\mathrm{N}$ load at a velocity of $1.4 \mathrm{~mm} / \mathrm{s}$ (ElectroForce 3200 , BOSE). This force and velocity was chosen based upon prior experiments showing that a $12-\mathrm{N}$ threshold force ruptured the ACL immediately on application of load and $1.4 \mathrm{~mm} / \mathrm{s}$ resulted in a midsubstance tear of the ligament (43). ACLr was identified through the waveform as a continued increase in displacement following release of the applied compressive force with an audible "popping" sound. Histology and x-ray analysis confirmed the rupture (data not shown). Contralateral knees served as unloaded controls. All mice received Buprecare (manufactured by Animalcare) $(0.05 \mathrm{mg} / \mathrm{kg})$ subcutaneously at the start of the experiment and moved freely after loading. All measurements were taken at days 0 (baseline), 1, 2, 3, 7, 14, and 21, unless stated otherwise. Day 21 was the predefined endpoint representing advanced/end stage arthritis for assessment of degeneration. Animals were excluded before this time only due to culling for welfare reasons or if there was no audible "pop" and change in displacement on loading ( $n=2$ for NBQX).

Following power calculations, pilot studies used 5 animals per group (detects differences of $50 \%-80 \%$ in degeneration in ACL rupture vs. uninjured controls), and the main study used 10 animals per group (detects differences in degeneration between ACLr+NBQX vs. ACLr+vehicle of 23\%-35\% with 80\% power).

NBQX treatment. A single i.a. injection of NBQX (20 mM in $10 \mu 1$ sterile water) was administered to 10 animals immediately following ACLr, while another 15 received an i.a. injection of vehicle (sterile water) immediately following ACLr. $20 \mathrm{mM}$ NBQX was chosen, as it had been reported to be safe in mice (45). Contralateral knees (intact ACL, $n=15$ ) were used as controls. To test dosing regimen, following ACLr, mice received either a single i.a. injection (day 0) of $20 \mathrm{mM}$ NBQX (or sterile water control), 2 injections (NBQX or vehicle, day 0 and 1), or 3 injections (NBQX or vehicle, day 0,1 , and 7) ( $n=5$ for each group). Contralateral knees (intact ACL with no load, $n=5$ ) and mice who received i.a. $20 \mathrm{mM} \mathrm{NBQX}$ without load (intact ACL, to assess effects of NBQX in normal joint) were used as controls.

Steroid and HA treatment. Steroid (Depo-Medrone, Pfizer, $10 \mathrm{mg} / \mathrm{kg}, n=7$ ), HA (Durolane, Bioventus, $8 \mathrm{mg} / \mathrm{kg}, n=8$ ), or vehicle control (saline, $n=7$ ) were administered as a single i.a. injection (10 $\mu 1) \mathrm{imme-}$ diately following ACLr. Depo-Medrone concentrations were of the same order of magnitude, taking into consideration SF volume (55-58), as those used clinically in the joints of dogs, horses, and humans $(59,60)$.

Sample processing. Human ACLs, menisci, and day 21 whole-mouse knees were fixed ( 2 days, 10\% neutral buffered formalin, MilliporeSigma), decalcified ( $4^{\circ} \mathrm{C}, 10 \%$ EDTA, Fisher Scientific), paraffin embedded, and coronally sectioned $(6 \mu \mathrm{m})$.

Histology. Consecutive sections from all animal experiments (day 21, numbers as above) were stained with H\&E (synovial inflammation) and toluidine blue/Safranin O (all manufactured by MilliporeSigma).

Two independent observers, blinded to treatment, used published scoring systems to assess mouse synovial inflammation (61) and knee degeneration (62) (Supplemental Tables 2 and 3) in 2 sections approximately $200-\mu \mathrm{m}$ apart. Average scores from both observers and both sections are presented.

Immunohistochemistry. GluRs were immunolocalized in sequential sections from human ACLs, menisci, and mouse knees (numbers as above) using antibodies to kainate receptor-1 (KA1) and AMPA receptor-2 (AMPAR2) (anti-KA1, ab67402; anti-iGluR2, ab52176; Abcam; see Supplemental Methods). Sections underwent antigen retrieval $(1 \mathrm{mg} / \mathrm{mL}$ trypsin, MilliporeSigma), peroxidase blocking, overnight incubation $\left(4^{\circ} \mathrm{C}\right)$ with primary antibody, and detection (Vectastain Elite $\mathrm{ABC}$ kit, nickel-enhanced diaminobenzidine, Vector Laboratories). Tris-buffered saline and IgG controls were included in every run.

To quantify positively staining cells in the ACL, 2-3 regions of interest were chosen (top, bottom, and middle of ACL, if possible), and the total cells and positive staining cells were counted. The number of positive staining cells were then presented as a percentage of total cells counted in the ACL.

Knee swelling and lameness. Mouse knee diameters were measured (mean of 3 readings per knee, Mitutoyo digital caliper, RS Components Ltd., observer blinded to treatment), and the data are presented as 
right minus left knee diameter. Lameness was scored on a scale of $0-10$, where 0 represents full weight bearing and no evidence of gait abnormality and 10 indicates nonweight bearing (adapted from ref. 63). Scores were averaged from 2 assessors blinded to treatment.

Glutamate ELISA. Glutamate concentrations were quantified in human SF (numbers as above) according to manufacturer's instructions (Glutamate ELISA kit KA1909, Novus Biologicals).

Statistics. Vehicle and drug treatments were compared using Minitab 18 software. Data were tested for normality and equal variances, followed by 2-sample, 2-tailed $t$ tests, 1-way ANOVA, or GLM 2-way or 3-way ANOVA with Tukey's post hoc tests. Nonparametric data used Kruskal-Wallis or Sheirer-Ray-Hare with Mann-Whitney post hoc tests. $P<0.05$ was considered significant. Mean \pm SD or box plots representing interquartile range, median, and all data points (including mean, indicated by crossed circle) are presented in the figures.

Study approval. Written informed consent was obtained from participants before inclusion in the study as per the research protocol, which was reviewed and approved by the ethics committee of Health and Care Research Wales (Wales REC 3, reference 10/MRE09/28, IRAS project 51853). Animal procedures were performed in compliance with the Animals (Scientific Procedures) Act 1986 (Home Office licenses 30/2959 and P287E87DF) according to the guidelines of Animal Research: Reporting of In Vivo Experiments and the Home Office, which approved these studies.

\section{Author contributions}

CSB designed research studies, conducted experiments, acquired data, analyzed data, and wrote the manuscript. SJG conducted experiments, acquired data, analyzed data, and edited the manuscript. EJB conducted experiments, acquired data, and edited the manuscript. ASW designed research studies, analyzed data, and edited the manuscript. DJM conceived and designed research studies, analyzed data, and wrote the manuscript.

\section{Acknowledgments}

This work was funded by Arthritis Research UK (20281) and the Medical Research Council Confidence in Concept and Life Sciences Bridging Fund Pathfinder Award (LSBF/R6-010) and used equipment, models, and human tissues provided by the Biomechanics and Bioengineering Centre Versus Arthritis (20781). We are grateful to Chris Wilson for provision of human tissue and clinical advice and Derek Scarborough for histology.

Address correspondence to: Deborah Mason, School of Biosciences, Sir Martin Evans Building, Cardiff University, Museum Avenue, Cardiff, CF10 3AX, Wales, United Kingdom. Phone: 44.0.2920874561; Email: Masondj@cardiff.ac.uk.

1. Woolf AD. Global burden of osteoarthritis and musculoskeletal diseases. BMC Musculoskelet Disord. 2015;16(Suppl 1):S3.

2. [No authors listed]. Arthritis. National Health Service. http://www.nhs.uk/conditions/arthritis/pages/introduction.aspx\#osteo. Accessed June 4, 2020.

3. [No authors listed]. NHS England Programme Budgeting Data (2012-3). National Health Service. http://www.england.nhs.uk/ resources/resources-for-ccgs/prog-budgeting/. Accessed June 4, 2020

4. Chen A, Gupte C, Akhtar K, Smith P, Cobb J. The global economic cost of osteoarthritis: how the UK compares. Arthritis. 2012;2012:698709.

5. Moradi-Lakeh M, et al. Burden of musculoskeletal disorders in the eastern mediterranean region, 1990-2013: findings from the Global Burden of Disease Study 2013. Ann Rheum Dis. 2017;76(8):1365-1373.

6. Kotlarz H, Gunnarsson CL, Fang H, Rizzo JA. Insurer and out-of-pocket costs of osteoarthritis in the US: evidence from national survey data. Arthritis Rheum. 2009;60(12):3546-3553.

7. Tang X, et al. The prevalence of symptomatic knee osteoarthritis in China Results from China Health and Retirement Longitudinal Study. Arthritis Rheumatol. 2016;68(3):648-653.

8. Brown TD, Johnston RC, Saltzman CL, Marsh JL, Buckwalter JA. Posttraumatic osteoarthritis: a first estimate of incidence, prevalence, and burden of disease. J Orthop Trauma. 2006;20(10):739-744.

9. Showery JE, Kusnezov NA, Dunn JC, Bader JO, Belmont PJ, Waterman BR. The rising incidence of degenerative and posttraumatic osteoarthritis of the knee in the united states military. J Arthroplasty. 2016;31(10):2108-2114.

10. Neuman P, Englund M, Kostogiannis I, Fridén T, Roos H, Dahlberg LE. Prevalence of tibiofemoral osteoarthritis 15 years after nonoperative treatment of anterior cruciate ligament injury: a prospective cohort study. Am J Sports Med. 2008;36(9):1717-1725.

11. Lohmander LS, Englund PM, Dahl LL, Roos EM. The long-term consequence of anterior cruciate ligament and meniscus injuries: osteoarthritis. Am J Sports Med. 2007;35(10):1756-1769.

12. Friel NA, Chu CR. The role of ACL injury in the development of posttraumatic knee osteoarthritis. Clin Sports Med. 2013;32(1):1-12. 
13. Hovelius L, Augustini BG, Fredin H, Johansson O, Norlin R, Thorling J. Primary anterior dislocation of the shoulder in young patients. A ten-year prospective study. J Bone Joint Surg Am. 1996;78(11):1677-1684.

14. Papandrea RF, Morrey BF, O'Driscoll SW. Reconstruction for persistent instability of the elbow after coronoid fracture-dislocation. J Shoulder Elbow Surg. 2007;16(1):68-77.

15. Weatherall JM, Mroczek K, McLaurin T, Ding B, Tejwani N. Post-traumatic ankle arthritis. Bull Hosp Jt Dis (2013). 2013;71(1):104-112

16. Lawrence RC, et al. Estimates of the prevalence of arthritis and selected musculoskeletal disorders in the United States. Arthritis Rheum. 1998;41(5):778-799.

17. Willett $\mathrm{NJ}$, et al. Intra-articular injection of micronized dehydrated human amnion/chorion membrane attenuates osteoarthritis development. Arthritis Res Ther. 2014;16(1):R47.

18. Heard BJ, et al. Single intra-articular dexamethasone injection immediately post-surgery in a rabbit model mitigates early inflammatory responses and post-traumatic osteoarthritis-like alterations. J Orthop Res. 2015;33(12):1826-1834.

19. Olson SA, Furman BD, Kraus VB, Huebner JL, Guilak F. Therapeutic opportunities to prevent post-traumatic arthritis: Lessons from the natural history of arthritis after articular fracture. J Orthop Res. 2015;33(9):1266-1277.

20. Furman BD, et al. Targeting pro-inflammatory cytokines following joint injury: acute intra-articular inhibition of interleukin-1 following knee injury prevents post-traumatic arthritis. Arthritis Res Ther. 2014;16(3):R134.

21. Wen ZH, Chang YC, Jean YH. Excitatory amino acid glutamate: role in peripheral nociceptive transduction and inflammation in experimental and clinical osteoarthritis. Osteoarthr Cartil. 2015;23(11):2009-2016.

22. Bonnet CS, Williams AS, Gilbert SJ, Harvey AK, Evans BA, Mason DJ. AMPA/kainate glutamate receptors contribute to inflammation, degeneration and pain related behaviour in inflammatory stages of arthritis. Ann Rheum Dis. 2015;74(1):242-251.

23. McNearney T, Speegle D, Lawand N, Lisse J, Westlund KN. Excitatory amino acid profiles of synovial fluid from patients with arthritis. J Rheumatol. 2000;27(3):739-745.

24. Lawand NB, McNearney T, Westlund KN. Amino acid release into the knee joint: key role in nociception and inflammation. Pain. 2000;86(1-2):69-74.

25. Jean YH, et al. Increased concentrations of neuro-excitatory amino acids in rat anterior cruciate ligament-transected knee joint dialysates: a microdialysis study. J Orthop Res. 2005;23(3):569-575.

26. Zhang GH, et al. The glutamatergic N-methyl-D-aspartate and non-N-methyl-D-aspartate receptors in the joint contribute to the induction, but not maintenance, of arthritic pain in rats. Neurosci Lett. 2003;351(3):177-180.

27. Tuboly G, et al. The inimitable kynurenic acid: the roles of different ionotropic receptors in the action of kynurenic acid at a spinal level. Brain Res Bull. 2015;112:52-60.

28. Gangadharan V, et al. Peripheral calcium-permeable AMPA receptors regulate chronic inflammatory pain in mice. J Clin Invest. 2011;121(4):1608-1623.

29. McNearney T, Baethge BA, Cao S, Alam R, Lisse JR, Westlund KN. Excitatory amino acids, TNF-alpha, and chemokine levels in synovial fluids of patients with active arthropathies. Clin Exp Immunol. 2004;137(3):621-627.

30. McNearney TA, et al. A peripheral neuroimmune link: glutamate agonists upregulate NMDA NR1 receptor mRNA and protein, vimentin, TNF-alpha, and RANTES in cultured human synoviocytes. Am J Physiol Regul Integr Comp Physiol. 2010;298(3):R584-R598.

31. Flood S, Parri R, Williams A, Duance V, Mason D. Modulation of interleukin-6 and matrix metalloproteinase 2 expression in human fibroblast-like synoviocytes by functional ionotropic glutamate receptors. Arthritis Rheum. 2007;56(8):2523-2534.

32. Wong PK, Campbell IK, Egan PJ, Ernst M, Wicks IP. The role of the interleukin-6 family of cytokines in inflammatory arthritis and bone turnover. Arthritis Rheum. 2003;48(5):1177-1189.

33. Lindblad SS, Mydel P, Hellvard A, Jonsson IM, Bokarewa MI. The N-methyl-d-aspartic acid receptor antagonist memantine ameliorates and delays the development of arthritis by enhancing regulatory T cells. Neurosignals. 2012;20(2):61-71.

34. Bailey AJ, Mansell JP. Do subchondral bone changes exacerbate or precede articular cartilage destruction in osteoarthritis of the elderly? Gerontology. 1997;43(5):296-304.

35. Buckland-Wright C. Subchondral bone changes in hand and knee osteoarthritis detected by radiography. Osteoarthr Cartil. 2004;12 Suppl A:S10-S19.

36. Hayami T, Pickarski M, Zhuo Y, Wesolowski GA, Rodan GA, Duong LT. Characterization of articular cartilage and subchondral bone changes in the rat anterior cruciate ligament transection and meniscectomized models of osteoarthritis. Bone. 2006;38(2):234-243

37. Mansell JP, Bailey AJ. Abnormal cancellous bone collagen metabolism in osteoarthritis. J Clin Invest. 1998;101(8):1596-1603.

38. Mansell JP, Tarlton JF, Bailey AJ. Biochemical evidence for altered subchondral bone collagen metabolism in osteoarthritis of the hip. Br J Rheumatol. 1997;36(1):16-19.

39. Grynpas MD, Alpert B, Katz I, Lieberman I, Pritzker KP. Subchondral bone in osteoarthritis. Calcif Tissue Int. 1991;49(1):20-26.

40. Li B, Aspden RM. Composition and mechanical properties of cancellous bone from the femoral head of patients with osteoporosis or osteoarthritis. J Bone Miner Res. 1997;12(4):641-651.

41. Thomsen JS, Straarup TS, Danielsen CC, Oxlund H, Brüel A. Relationship between articular cartilage damage and subchondral bone properties and meniscal ossification in the Dunkin Hartley guinea pig model of osteoarthritis. Scand J Rheumatol. 2011;40(5):391-399.

42. Johnson DL, Urban WP, Caborn DN, Vanarthos WJ, Carlson CS. Articular cartilage changes seen with magnetic resonance imaging-detected bone bruises associated with acute anterior cruciate ligament rupture. Am J Sports Med. 1998;26(3):409-414.

43. Gilbert SJ, Bonnet CS, Stadnik P, Duance VC, Mason DJ, Blain EJ. Inflammatory and degenerative phases resulting from anterior cruciate rupture in a non-invasive murine model of post-traumatic osteoarthritis. J Orthop Res. 2018;36(8):2118-2127.

44. Christiansen BA, Anderson MJ, Lee CA, Williams JC, Yik JH, Haudenschild DR. Musculoskeletal changes following non-invasive knee injury using a novel mouse model of post-traumatic osteoarthritis. Osteoarthr Cartil. 2012;20(7):773-782.

45. Dalgaard L, Hjortkjaer RK, Regnier B, Nordholm L. Pharmacokinetics of the neuroprotective glutamate antagonist NBQX (6-nitro-7-sulfamoyl-benzo(f)quinoxaline-2,3-dione) in mice, rats, and dogs. Interactions with probenecid. Drug Metab Dispos. 1994;22(2):289-293. 
46. Elsaid KA, Zhang L, Shaman Z, Patel C, Schmidt TA, Jay GD. The impact of early intra-articular administration of interleukin-1 receptor antagonist on lubricin metabolism and cartilage degeneration in an anterior cruciate ligament transection model. Osteoarthr Cartil. 2015;23(1):114-121.

47. Larsen C, et al. Intra-articular depot formulation principles: role in the management of postoperative pain and arthritic disorders. J Pharm Sci. 2008;97(11):4622-4654.

48. Wernecke C, Braun HJ, Dragoo JL. The effect of intra-articular corticosteroids on articular cartilage: a systematic review. Orthop J Sports Med. 2015;3(5):2325967115581163.

49. Bajpayee AG, et al. Sustained intra-cartilage delivery of low dose dexamethasone using a cationic carrier for treatment of post traumatic osteoarthritis. Eur Cell Mater. 2017;34:341-364.

50. Jüni P, et al. Intra-articular corticosteroid for knee osteoarthritis. Cochrane Database Syst Rev. 2015;(10):CD005328.

51. Herrling P, ed. Excitatory Amino Acids: Clinical Results with Antagonists. Cambridge, MA: Academic Press; 1997.

52. Little CB, Zaki S. What constitutes an "animal model of osteoarthritis"--the need for consensus? Osteoarthr Cartil. 2012;20(4):261-267.

53. Kilkenny C, Browne WJ, Cuthill IC, Emerson M, Altman DG. Improving bioscience research reporting: the ARRIVE guidelines for reporting animal research. PLoS Biol. 2010;8(6):e1000412.

54. Poulet B, Hamilton RW, Shefelbine S, Pitsillides AA. Characterizing a novel and adjustable noninvasive murine joint loading model. Arthritis Rheum. 2011;63(1):137-147.

55. Kraus VB, Stabler TV, Kong SY, Varju G, McDaniel G. Measurement of synovial fluid volume using urea. Osteoarthr Cartil. 2007;15(10):1217-1220.

56. Seifer DR, Furman BD, Guilak F, Olson SA, Brooks SC, Kraus VB. Novel synovial fluid recovery method allows for quantification of a marker of arthritis in mice. Osteoarthr Cartil. 2008;16(12):1532-1538.

57. Anirudh A, Ranganath L. Synovial fluid analysis in dogs with elbow, hip and stifle joint disorders. Int J Appl Pure Sci Agric. 2015;1(7):07-2015

58. Van Pelt RW. Characteristics of normal equine tarsal synovial fluid. Can J Comp Med Vet Sci. 1967;31(12):342-347.

59. [No authors listed]. Depo-Medrone 40mg/mL PL 00057/0963. DataPharm. https://www.medicines.org.uk/emc/product/8957/ smpc. Accessed June 4, 2020.

60. [No authors listed]. Depo-Medrone V $40 \mathrm{mg} / \mathrm{mL}$ Suspension for Injection. NOAH Compendium. http://www.noahcompendium.co.uk/?id=-456912. Accessed June 4, 2020

61. Nowell MA, et al. Therapeutic targeting of IL-6 trans signaling counteracts STAT3 control of experimental inflammatory arthritis. J Immunol. 2009;182(1):613-622.

62. Glasson SS, Chambers MG, Van Den Berg WB, Little CB. The OARSI histopathology initiative - recommendations for histological assessments of osteoarthritis in the mouse. Osteoarthr Cartil. 2010;18 Supp1 3:S17-S23

63. Pfeiffenberger U, et al. Assessment and refinement of intra-bone marrow transplantation in mice. Lab Anim. 2015;49(2):121-131. 